\section{Commentary on: Evaluation of an NHS practice- based minor oral surgery service}

\author{
T. A. Dyer and A. C. Dhamija
}

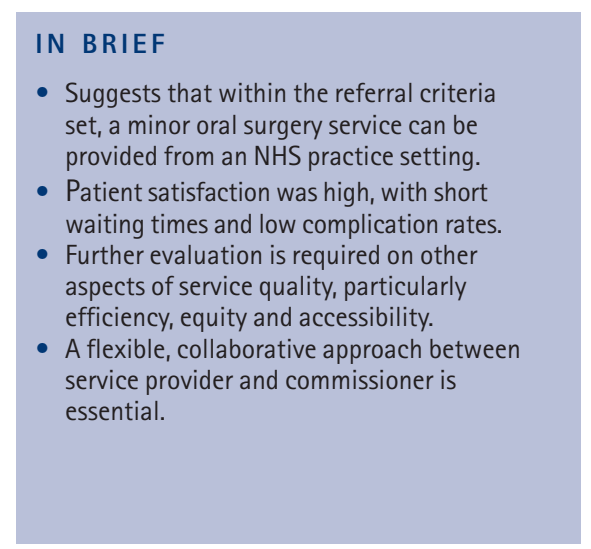

\begin{abstract}
Objectives To evaluate a pilot specialist practice-based minor oral surgery service. Methods Service monitoring data were analysed to evaluate activity, waiting times and outcomes. Patient satisfaction was assessed by a modified version of the Dental Visit Satisfaction Scale. Results 0 f 705 treatment appointments, 12.7\% were not attended and treatment was not provided in another $11.6 \%$. Mean waiting time from referral receipt to treatment was 6.8 weeks. Treatment provided included surgical removal of third molars, surgical removal of non-third molars and surgical endodontics $(26.7 \%, 60.4 \%$ and $4.9 \%$ of cases, respectively). Antibiotics were prescribed in $15.0 \%$ of treatment cases and $2.3 \%$ of treatment cases required appointments for postoperative complications. The response rate for the satisfaction survey was $81 \%$. All participants reported overall satisfaction and strongly agreed/agreed with positive attitudinal statements about the oral surgeon's communication/information giving, technical competence and understanding/acceptance. $77.8 \%$ were seen on time and 22.2\% within 15 minutes of their appointment. Overall 74.1\% felt the standard of service was better than they would expect from a hospital and none felt it was worse. Conclusion The findings of the evaluation suggest that specialist minor oral surgery can be successfully provided in dental practice and is acceptable to patients.
\end{abstract}

\section{COMMENTARY}

The introduction of the new dental contract gives primary care organisations (PCOs) opportunities to locally commission primary care services including specialist services, which potentially offer more efficient and accessible services to those in secondary care. This paper is timely in its reporting of a service evaluation comprising an audit of activity, waiting times and patient satisfaction of an NHS practice-based specialist minor oral surgery service.

Over a 12 month pilot period, 705 patients were referred by 51 local GDPs, resulting in 513 surgical procedures. The mean waiting time to first treatment appointment was 6.8 weeks and of those, $77.0 \%$ were treated in one appointment. A small percentage (4.4\%) required more than two appointments or reviews. The majority of procedures (60.4\%) were for surgical removal of non-third molars, followed by surgical removal of third molars (26.7\%). Of those (97) not treated at first appointment, 20.6\% were reported as inappropriate referrals and
$12.4 \%$ required referral to secondary care for $\mathrm{GA} /$ sedation services.

The qualitative aspects of the service were investigated through 100 patient questionnaires with a response rate of $81 \%$. In summary, the majority of clients reported very positive views and satisfaction regarding affective behaviour, technical competence and efficiency of the service.

This paper identifies a demand for commissioned specialist oral surgery services in primary care and demonstrates an acceptable clinical referral pathway and treatment provision. The authors highlight some limitations of the audit and identify areas for further enquiry, in particular the reasons for a failure rate of $12.7 \%$ and for non-treatment at first appointment (11.6\%). Efficiency of the service could be increased by improving both of these factors through stricter application of referral criteria and improved patient assessment.

The paper does not explore the issues of governance and PCOs will require both clinical and quality assurances from such services. It may therefore be important to ensure that these services operate as part of a managed clinical network including commissioners and clinicians from both primary and secondary care. In this way efficient, acceptable and integrated pathways will result in the most appropriate setting for the patient.

K. Klause
Consultant in Dental Public Health,
Wirral

DOI: 10.1038/sj.bdj.2009.1157 